\title{
An Analysis of a Partial Temporary Immunity SIR Epidemic Model with Nonlinear Treatment Rate
}

\author{
Saba Noori Majeed ${ }^{1^{*}}$ \\ Raid Kamel Naji ${ }^{2}$
}

Received 25/3/2018, Accepted 7/3/2019, Published 1/9/2019

This work is licensed under a Creative Commons Attribution 4.0 International License.

\begin{abstract}
:
A partial temporary immunity SIR epidemic model involv nonlinear treatment rate is proposed and studied. The basic reproduction number $R_{0}$ is determined. The local and global stability of all equilibria of the model are analyzed. The conditions for occurrence of local bifurcation in the proposed epidemic model are established. Finally, numerical simulation is used to confirm our obtained analytical results and specify the control set of parameters that affect the dynamics of the model.
\end{abstract}

Key words: Nonlinear treatment rate, Partial temporary immunity, SIR epidemic model.

\section{Introduction:}

In biology, immunity means resistance of an organism to infection or disease. Many researchers have studied immunity, in medicine and biology. Mathematically epidemic models represent a useful tool to analyze this subject, and reach all factors affecting it through mathematical equations to predict their behavior (1). In general, immunity to the diseases is divided into two types known as lifelong immunity and temporary immunity. Polio is one of the diseases that caused lifelong immunity. In fact Bunimovich-Mendrazitsky and Stone (2) studied Polio as an epidemic model in order to explain the disease development. D'Onofrio in (3) studied Pulse vaccination strategy in the SIR epidemic model to verify the use of a pulse vaccination strategy to eradicate infectious diseases. However, Dubey et. al (4), investigated an SIR Model with nonlinear incidence and treatment rates, they showed that effective treatment and hence increasing the number of individuals who have lifelong ammunition, the resource limitation of treatments should be minimized. On the other hand, there are diseases cause temporary immunity, the subject that our research deals with for instance bacteria that causes Pertussis. Many researchers studied temporary immunity such as Taylor and Carr (5), who studied temporary immunity in an SIR-based model with delayed coupling between the susceptible and removed classes. Sahu and Dhar

${ }^{T}$ Department of Computer Science, College of Education for Women, University of Baghdad, Baghdad, Iraq

2 Department of Mathematics, College of Science, University of Baghdad, Baghdad, Baghdad, Iraq

*Corresponding author: snoori39@yahoo.com
(6), studied in their paper an SVEIS epidemic model for an infectious disease spreads in the host population through horizontal transmission and the role that temporary immunity plays in the spread of disease. Accordingly, in this paper an SIR epidemic model with temporary immunity and gathering it to nonlinear treatment rate is proposed and studied. The treatment function is taken as a modified Holling type II. Recently Naji and Hussien (7) have proposed and investigated a mathematical model that involves two different types of infectious diseases that spread in the host population horizontally as well as vertically. Keeping the above review in view, in this paper the objective is to understand the effects of treatment rate on the spread of disease. This paper is organized as following. Section 2, deals with the formulation of the model and discusses the existence, uniqueness, and boundedness of their solution. In section 3, the existence of the equilibrium points and their stability analyses are carried out. The local bifurcation analysis is studied in section 4 . While the numerical simulation and the discussion is presented in section 5 .

\section{Mathematical Model:}

It is well known that the study of the epidemic systems is very important subject for the human and all other species due to the impact of the infectious diseases on their existence in the environment. Accordingly, in this section an epidemic system of SIR type of disease with partial temporary immunity and saturated treatment function are proposed and studied. This type of disease divides the population to three mutual compartments 
namely $S(t), I(t)$ and $R(t)$, where $S(t)$ represents the number of individuals in the susceptible compartment at time $t, I(t)$ represents the number of individuals in the infected compartment at time $t$, while $R(t)$ is dented to the number of individuals in the removal compartment at time $t$. Consequently in order to formulate the dynamical system that simulates the dynamics of such an epidemic system the following hypotheses are adopted:

1. All the new born individuals are susceptible and the system supplied by a constant number of susceptible individuals with recruitment rate $A>0$.

2. The disease is transmitted from infected individuals to susceptible individuals by direct contact between them according to mass action law or indirect way due to contaminated water or air or others with direct infection rate $\alpha>0$ and indirect infection rate $\alpha_{1} \geq 0$ respectively. Further, the disease may causes death for the infected individuals with disease death rate $\delta_{1}>0$.

3. The infected individuals recover from the disease and are transferred to the removal compartment depending on the individual natural immunity with natural recovery rate $\delta_{2}>0$ or due to treatment procedure with nonlinear treatment rate given by $\frac{a I}{1+b I}$, see (8), where $a>0$ represents the maximum treatment rate while $b>0$ represents the resource limitation.

4. The immunity gained by recovered individuals is temporary and hence portion of the recovered individuals will be transferred again to the susceptible individuals with partial temporary immunity rate $\delta_{3}>0$.

5. Finally, each individuals in the population faces a natural death with death rate given by $\delta_{0}>0$.

Therefore, the dynamics of above described epidemic system can be described by the following set of differential equations:

$$
\begin{aligned}
& \frac{d S}{d t}=A-\delta_{0} S-\alpha S I-\alpha_{1} S+\delta_{3} R \\
& \frac{d I}{d t}=\alpha S I-\delta_{0} I-\delta_{1} I-\delta_{2} I-\frac{a I}{1+b I}+\alpha_{1} S \\
& \frac{d R}{d t}=\delta_{2} I-\delta_{0} R-\delta_{3} R+\frac{a I}{1+b I}
\end{aligned}
$$

with an initial condition in the region $\mathbb{R}_{+}^{\mathbf{3}}=$ $\left\{(S, I, R) \in \mathbb{R}_{+}^{3} ; S>0, I \geq 0, R \geq 0\right\} ; \quad$ clearly the interaction functions given on the right hand side of system (1) are continuously differentiable. Therefore, the solution of system (1) with nonnegative initial condition exists and is unique. Moreover the solution is uniformly bounded as shown in the following theorem.
Theorem 1. All the solutions of system (1), which are initiate in the region $\mathbb{R}_{+}^{3}$ are uniformly bounded. Proof. Let $N(t)=S(t)+I(t)+R(t)$ represents the population size, then

$$
\frac{d N}{d t}=\frac{d S}{d t}+\frac{d I}{d t}+\frac{d R}{d t} \leq A-\delta_{0} N
$$

Now by solving the above linear differential inequality we obtain that

$$
N(t) \leq N(0) e^{-\delta_{0} t}+\frac{A}{\delta_{0}}\left(1-e^{-\delta_{0} t}\right),
$$

Thus, for $t \rightarrow \infty$ it is obtained $N(t) \leq \frac{A}{\delta_{0}}$, hence, all the solutions of system (1) initiate in $\mathbb{R}_{+}^{3}$ are uniformly bounded.

Note that theorem (1) shows that all solutions of the model are non-negative and bounded, thus the model is well behaved biologically.

\section{Existence of Equilibrium Points and Their Stability}

It is easy to verify that system (1) has at most two biologically feasible equilibrium points, namely $E_{0}$ and $E_{1}$, which are known as the free disease equilibrium point and the endemic equilibrium point respectively. The existence conditions and their stability conditions are established below. In case of the disappearance of the disease from the environment then the population will contains only susceptible individuals and hence the so called disease free equilibrium point $E_{0}=\left(S_{0}, 0,0\right)=$ $\left(\frac{A}{\delta_{0}}, 0,0\right)$

exists provided that the indirect infection rate is zero $\alpha_{1}=0$.

However, in case of the existence of disease in the environment then all the compartments re exist and hence the so called endemic equilibrium point $E_{1}=\left(S_{1}, I_{1}, R_{1}\right)$, which given by

$$
\begin{aligned}
& S_{1}=\left[\frac{\left(\delta_{0}+\delta_{1}+\delta_{2}\right)\left(1+b I_{1}\right)+a}{\left(1+b I_{1}\right)\left(\alpha_{1}+\alpha I_{1}\right)}\right] I_{1} \\
& R_{1}=\left[\frac{a+\delta_{2}\left(1+b I_{1}\right)}{\left(\delta_{0}+\delta_{3}\right)\left(1+b I_{1}\right)}\right] I_{1}
\end{aligned}
$$

while $I_{1}$ is a positive root to the following cubic equation

$$
\begin{aligned}
& \qquad D_{1} I_{1}^{3}+D_{2} I_{1}^{2}+D_{3} I_{1}+D_{4}=0 \\
& \text { here } \quad D_{1}=-\alpha b\left[\delta_{0}\left(\delta_{0}+\delta_{1}+\delta_{2}\right)+\right. \\
& \left.\delta_{3}\left(\delta_{0}+\delta_{1}\right)\right]
\end{aligned}
$$

$$
\begin{gathered}
D_{2}=b\left(\delta_{0}+\delta_{3}\right)\left[\begin{array}{c}
\alpha A-\left(\delta_{0}+\delta_{1}+\delta_{2}\right)\left(\delta_{0}+\alpha_{1}\right)- \\
\frac{\alpha}{b}\left(\delta_{0}+\delta_{1}+\delta_{2}+a\right)
\end{array}\right] \\
+\delta_{3}\left[\alpha\left(\delta_{2}+a\right)+b \alpha_{1} \delta_{2}\right] \\
D_{3}=\left(\delta_{0}+\delta_{3}\right)\left[A\left(\alpha_{1} b+\alpha\right)-\right. \\
\left.\left(\delta_{0}+\delta_{1}+\delta_{2}+a\right)\left(\delta_{0}+\alpha_{1}\right)\right]+\alpha_{1} \delta_{3}\left(\delta_{2}+a\right) \\
D_{4}=\alpha A\left(\delta_{0}+\delta_{3}\right)
\end{gathered}
$$


exists uniquely in the interior of positive octant provided that the following condition, which is obtained using Discard's rule (9), is satisfied:

$$
D_{2}<0 \text { OR } D_{3}>0
$$

Now before we start our study the stability of the above equilibrium points, the basic reproduction number $R_{0}$, which represents the number of new infected people caused by a single infected patient when introduced into a completely susceptible population, is computed. It is well known that the value of this number plays a vital role in the stability of the system (1). According to definition of basic reproduction number which is given in (10), rewrite system (1) in the form:

$x^{\prime}=\mathcal{H}(x)-\mathcal{M}(x)$

where $x=(I, S, R)^{T}, \mathcal{H}(x)$ is the matrix of new infection individuals and $\mathcal{M}(x)$ is the matrix of transferred individuals between the infected compartments and out of the infected compartments. Therefore

$$
\begin{gathered}
\mathcal{H}(x)=\left[\begin{array}{c}
\alpha S I+\alpha_{1} S \\
0 \\
0
\end{array}\right] ; \\
\mathcal{M}(x)=\left[\begin{array}{c}
\delta_{0} I+\delta_{1} I+\delta_{2} I+\frac{a I}{1+b I} \\
\delta_{0} S+\alpha S I+\alpha_{1} S-\delta_{3} R-A \\
\delta_{0} R+\delta_{3} R-\frac{a I}{1+b I}-\delta_{2} I
\end{array}\right] .
\end{gathered}
$$

So the derivative of $\mathcal{H}(x)$ and $\mathcal{M}(x)$, with respect to vector $x$, at the disease free equilibrium point $E_{0}$ are computed as follow:

$D \mathcal{H}(\mathrm{x})=\left[\begin{array}{ccc}\frac{\alpha A}{\delta_{0}} & 0 & 0 \\ 0 & 0 & 0 \\ 0 & 0 & 0\end{array}\right]=\left[\begin{array}{cc}H & \mathbf{0} \\ \mathbf{0} & \mathbf{0}\end{array}\right] ; \quad D \mathcal{M}(x)=$ $\left[\begin{array}{ccc}\delta_{0}+\delta_{1}+\delta_{2}+a & 0 & 0 \\ \frac{\alpha A}{\delta_{0}} & \delta_{0} & -\delta_{3} \\ -a-\delta_{2} & 0 & \delta_{0}+\delta_{3}\end{array}\right]=\left[\begin{array}{cc}M & 0 \\ \boldsymbol{j}_{1} & \boldsymbol{j}_{2}\end{array}\right]$,

where $H=\left[\frac{\alpha A}{\delta_{0}}\right] ; \quad$ and $M=\left[\delta_{0}+\delta_{1}+\delta_{2}+a\right]$. Consequently, $R_{0}$ is equal to spectral radius of new generation matrix $\mathcal{H} \mathcal{M}^{-1}$. Therefore, the reproduction number of system (1) is given by

$$
R_{0}=\rho\left(\mathcal{H} \mathcal{M}^{-1}\right)=\frac{\alpha A}{\delta_{0}\left(\delta_{0}+\delta_{1}+\delta_{2}+a\right)}
$$

Theorem 2: The disease free equilibrium point of system (1) is locally asymptotically stable provided that condition (7a) holds, while its unstable under condition (7b). $R_{0}<1$

$$
R_{0}>1
$$

Proof. The Jacobian matrix of system (1) at $E_{0}$ can be written as:

$$
\begin{gathered}
J\left(E_{0}\right)=\left[b_{i j}\right]_{3 \times 3}= \\
{\left[\begin{array}{ccc}
-\delta_{0} & \frac{-\alpha A}{\delta_{0}} & \delta_{3} \\
0 & \frac{\alpha A}{\delta_{0}}-\left(\delta_{0}+\delta_{1}+\delta_{2}+a\right) & 0 \\
0 & \delta_{2}+a & -\left(\delta_{0}+\delta_{3}\right)
\end{array}\right]}
\end{gathered}
$$

(8a)

Then the characteristic equation of $J\left(E_{0}\right)$ is given by:

$$
\left(-\delta_{0}-\lambda\right)\left(\lambda^{2}+\mathrm{A}_{1} \lambda+\mathrm{A}_{2}\right)=0
$$

where $\mathrm{A}_{1}=-\left[\frac{\alpha A}{\delta_{0}}-\left(\delta_{0}+\delta_{1}+\delta_{2}+a\right)-\left(\delta_{0}+\right.\right.$

$\left.\left.\delta_{3}\right)\right]$

$$
\mathrm{A}_{2}=-\left(\alpha_{1}+\delta_{0}\right)\left(\frac{\alpha A}{\delta_{0}}-\left(\delta_{0}+\delta_{1}+\delta_{2}+a\right)\right)
$$

Obviously, it is easy to verify that condition (7a) guarantees that $A_{1}>0$ and $A_{2}>0$. Therefore according to Routh-Hurwitz criterion (11), the second order polynomial in Eq. (8b) has two roots (eigenvalues for $E_{0}$ ) with negative real parts. Further, since the third eigenvalue is given by $\lambda=-\delta_{0}<0$, hence the disease free equilibrium point $E_{0}$ is locally asymptotically stable. On the other hand condition ( $7 \mathrm{~b}$ ) leads to $A_{2}<0$, and then the second order polynomial in Eq. (8b) has two roots with opposite signs. Hence, $E_{0}$ is an unstable saddle point, which completes the proof.

Theorem 3: The endemic equilibrium point $E_{1}=\left(S_{1}, I_{1}, R_{1}\right)$ of system (1) is locally asymptotically stable, provided that

$$
\begin{aligned}
& \left(\frac{\delta_{3}}{\left(\delta_{0}+\delta_{3}\right)}\right)\left[\frac{\delta_{2}\left(1+b I_{1}\right)^{2}+a}{\left(1+b I_{1}\right)^{2}}\right]<\alpha S_{1}< \\
& \frac{\left(\left(\delta_{0}+\delta_{1}+\delta_{2}\right)\left(1+b I_{1}\right)^{2}+a\right)}{\left(1+b I_{1}\right)^{2}}
\end{aligned}
$$

Proof. The Jacobian matrix of system (1) at $E_{1}$ can be written as

$$
\begin{aligned}
& J\left(E_{1}\right)= \\
& {\left[\begin{array}{ccc}
-\left(\delta_{0}+\alpha I_{1}+\alpha_{1}\right) & -\alpha S_{1} & \delta_{3} \\
\alpha I_{1}+\alpha_{1} & \alpha S_{1}-\left(\delta_{0}+\delta_{1}+\delta_{2}\right)-\frac{a}{\left(1+b I_{1}\right)^{2}} & 0 \\
0 & \delta_{2}+\frac{a}{\left(1+b I_{1}\right)^{2}} & -\left(\delta_{0}+\delta_{3}\right)
\end{array}\right]}
\end{aligned}
$$

(10a)

Then the characteristic equation of $E_{1}$ is given by:

$$
\lambda^{3}+\Omega_{1} \lambda^{2}+\Omega_{2} \lambda+\Omega_{3}=0
$$

here $\Omega_{1}=-\left(c_{11}+c_{22}+c_{33}\right), \quad \Omega_{2}=c_{11} c_{22}-$ $c_{12} c_{21}+c_{11} c_{33}+c_{22} c_{33}$ and $\Omega_{3}=c_{21}\left(c_{12} c_{33}-\right.$ $\left.c_{32} c_{13}\right)-c_{11} c_{22} c_{33}$, where $c_{i j}$ represent the coefficients of the Jacobian matrix given by (10a).

Furthermore 


$$
\begin{gathered}
\Delta=\Omega_{1} \Omega_{2}-\Omega_{3}=\left(c_{12} c_{21}-c_{11} c_{22}\right)\left(c_{11}+c_{22}\right)-c_{11} c_{33}\left(c_{11}+c_{33}\right) \\
-c_{22} c_{33}\left(c_{22}+c_{33}\right)-2 c_{11} c_{22} c_{33}+c_{21} c_{13} c_{32}
\end{gathered}
$$

According to Routh-Hurwitz criterion, $E_{1}$ is locally asymptotically stable provided that $\Omega_{1}>0, \Omega_{3}>0$ and $\Delta>0$. Now it is easy to verify that all these conditions are satisfied provided that condition (9) holds, and hence the proof is complete.

Theorem 4: Assume that the disease free equilibrium point of system (1) is locally asymptotically stable then it is globally asymptotically stable provided that:

$$
\frac{\alpha A}{\delta_{0}}<\left(\delta_{0}+\delta_{1}\right)
$$

Proof.Let $\mathcal{W}_{1}=\left(S-S_{0}-S_{0} \ln \frac{S}{S_{0}}\right)+I+R$,

clearly $\mathcal{W}_{1}: \mathbb{R}_{+}^{3} \rightarrow \mathbb{R}$ is continuously differential function such that $\mathcal{W}_{1}\left(S_{0}, 0,0\right)=0$, and $\mathcal{W}_{1}(S, I, R)>0 ; \forall(S, I, R) \neq\left(S_{0}, 0,0\right)$.

Differentiating $\mathcal{W}_{1}$ with respect to time and then after doing some algebraic computation, we have:

$\frac{d \mathcal{W}_{1}}{d t} \leq-\frac{\delta_{0}}{S}\left(S-S_{0}\right)^{2}-\left(\left(\delta_{0}+\delta_{1}\right)-\alpha S_{0}\right) I-\delta_{0} R$

Clearly condition (11) agree with $R_{0}<1$ and guarantee that $\frac{d \mathcal{W}_{1}}{d t}<0$. Hence according to Laypunov second method of stability the free disease equilibrium point $E_{0}$ is locally

Next theorem establishes the global stability conditions for the endemic equilibrium point of system (1).

Theorem 5: Assume that the endemic equilibrium point $E_{1}$, of system (1) is locally asymptotically stable, then its globally asymptotically stable in subregion that satisfies the following conditions:

$$
\alpha S_{1}<\frac{\left(\left(\delta_{0}+\delta_{1}+\delta_{2}\right)\left(1+b I_{1}\right)(1+b I)+a\right)}{\left(1+b I_{1}\right)(1+b I)}
$$

$$
\begin{aligned}
& q_{12}^{2}<q_{11} q_{22} \\
& {q_{13}}^{2}<q_{11} q_{33} \\
& {q_{23}}^{2}<q_{22} q_{33}
\end{aligned}
$$

where $q_{i j}$ is given in the proof.

Proof. Consider the following function $\mathcal{W}_{2}=$ $\frac{\left(S-S_{1}\right)^{2}}{2}+\frac{\left(I-I_{1}\right)^{2}}{2}+\frac{\left(R-R_{1}\right)^{2}}{2}$. Clearly we have $\mathcal{W}_{2}=\mathbb{R}_{+}^{3} \stackrel{2}{\rightarrow}$ is continuously differential function with $\mathcal{W}_{2}\left(S_{1}, I_{1}, R_{1}\right)=0$, while $\mathcal{W}_{2}(S, I, R)>$ $0 ; \forall(S, I, R) \neq\left(S_{1}, I_{1}, R_{1}\right)$. Moreover, straightforward computation gives that:

$$
\begin{aligned}
& \text { here } \quad q_{11}=\delta_{0}+\alpha_{1}+\alpha I, \quad q_{12}=\alpha_{1}+\alpha I- \\
& \begin{array}{cl}
\alpha S_{1}, \quad q_{13}=\delta_{3}, q_{33}=\delta_{0}+\delta_{3} \\
\\
q_{22}=\delta_{0}+\delta_{1}+\delta_{2}+\frac{a}{(1+b I)\left(1+b I_{1}\right)}-\alpha S_{1}, \\
q_{23}= & \delta_{2}+\frac{a}{(1+b I)\left(1+b I_{1}\right)} .
\end{array}
\end{aligned}
$$

Thus according to the given conditions it's easy to verify that

$$
\begin{aligned}
\frac{d W_{2}}{d t} \leq- & \left(\sqrt{\frac{q_{11}}{2}}\left(S-S_{1}\right)-\sqrt{\frac{q_{22}}{2}}\left(I-I_{1}\right)\right)^{2} \\
& -\left(\sqrt{\frac{q_{22}}{2}}\left(I-I_{1}\right)-\sqrt{\frac{q_{33}}{2}}\left(R-R_{1}\right)\right)^{2} \\
& -\left(\sqrt{\frac{q_{11}}{2}}\left(S-S_{1}\right)-\sqrt{\frac{q_{33}}{2}}\left(R-R_{1}\right)\right)^{2}
\end{aligned}
$$

Clearly, $\frac{d \mathcal{W}_{2}}{d t}<0$ and hence due to the second Lyapunov theorem the endemic equilibrium point is globally asymptotically stable and the proof is complete

\section{Local Bifurcation Analysis}

In this section, the local bifurcation (such as saddle-node, transcritical and pitchfork) is studied to find out the influence of changing one of the parameters value, on the dynamical behavior of system (1) around the disease free equilibrium point $E_{0}$. An application of the Sotomayor's theorem of local bifurcation (11) is carried out in order to specify the type of bifurcation near the equilibrium point as shown in the following theorem.

Theorem 6: Assume that $R_{0}=1$, then system (1) undergoes a transcrtical bifurcation near the disease free equilibrium point $E_{0}$, but neither saddle-node bifurcation nor pitchfork bifurcation can occur, provided that

$$
\alpha^{*} z+a b q \neq 0
$$

where $z$ and $q$ are given in the proof.

Proof. It is easy to verify that $R_{0}=1$ is an equivalent to $\alpha^{*}=\frac{\delta_{0}\left(\delta_{0}+\delta_{1}+\delta_{2}+a\right)}{A}$ (other parameter

could be chosen too). Now by substituting the value of $\alpha^{*}$ in the Jacobian matrix given by (8a) we obtain that:

$$
\begin{gathered}
J=D f\left(E_{0} \cdot \alpha^{*}\right)= \\
{\left[\begin{array}{ccc}
-\delta_{0} & \frac{-\alpha^{*} A}{\delta_{0}} & \delta_{3} \\
0 & 0 & 0 \\
0 & \delta_{2}+a & -\left(\delta_{0}+\delta_{3}\right)
\end{array}\right]}
\end{gathered}
$$

$$
\begin{aligned}
& \frac{d \mathcal{W}_{2}}{d t}=-q_{11}\left(S-S_{1}\right)^{2}-q_{22}\left(I-I_{1}\right)^{2}-q_{33}\left(R-R_{1}\right)^{2} \\
& \quad+q_{12}\left(S-S_{1}\right)\left(I-I_{1}\right)+q_{13}\left(S-S_{1}\right)(R-R)+q_{23}\left(I-I_{1}\right)\left(R-R_{1}\right)
\end{aligned}
$$


Clearly the second eigenvalue of $J, \lambda_{I}$ in the Idirection is zero $\left(\lambda_{I}=0\right)$, while $\lambda_{s}=-\delta_{0}$ and

$\lambda_{R}=-\left(\delta_{0}+\delta_{3}\right)$. Hence the disease free equilibrium point is a non-hyperbolic point for $R_{0}=1$. Further the eigenvector, say $K=$ $\left(k_{1}, k_{2}, k_{3}\right)^{T}$, that corresponds $\lambda_{I}=0$ is determined as: $\quad K=\left(z k_{3}, q k_{3}, k_{3}\right)^{T}$

where $\quad z=-\frac{\left(\delta_{0}+\delta_{1}+\delta_{2}+a\right) \delta_{0}+\left(\delta_{0}+\delta_{1}\right) \delta_{3}}{\delta_{0}\left(\delta_{2}+a\right)}, q=\frac{\delta_{0}+\delta_{3}}{\delta_{2}+a}$ and $k_{3}$ is a non-zero real number.

Similarly the eigenvector $W=\left(\begin{array}{lll}w_{1} & w_{2} & w_{3}\end{array}\right)^{T}$ that corresponds $\lambda_{I}=0$ of $J^{T}$ is determined as:

$$
W=\left(0, w_{2}, 0\right)^{T}
$$

where $w_{2}$ is a non-zero real number. Now, rewrite system (1) as vector form $\frac{d X}{d t}=f(x)$, where $X=(S, I, R)^{T}$ and $f=\left(f_{1}, f_{2}, f_{3}\right)^{T}$ be the interaction functions vector given in system (1). Then by determining $\frac{d f}{d \alpha}=f_{\alpha}$, we get that:

$$
f_{\alpha}=\left[\begin{array}{c}
-S I \\
S I \\
0
\end{array}\right] \text {, then we get } f_{\alpha}\left(E_{0}, \alpha^{*}\right)=\left[\begin{array}{l}
0 \\
0 \\
0
\end{array}\right]
$$

Therefore: $w^{T} \cdot f_{\alpha}\left(E_{0}, \alpha^{*}\right)=0$, consequently, by using Sotomayor theorem, system (1) has no saddlenode bifurcation near $E_{0}$ and $\alpha=\alpha^{*}$. Now in order to investigate the other types of bifurcation, the derivative of $f_{\alpha}$ with respect to vector $X$ say $D f_{\alpha}\left(E_{0}, \alpha^{*}\right)$ is computed:

$$
D f_{\alpha}\left(E_{0}, \alpha^{*}\right)=\left[\begin{array}{ccc}
0 & -S_{0} & 0 \\
0 & S_{0} & 0 \\
0 & 0 & 0
\end{array}\right] \text {, }
$$

So this gives that $W^{T}\left[D f_{\alpha}\left(E_{0}, \alpha^{*}\right), K\right]=$ $w_{2} q k_{3} S_{0} \neq 0$. Again, in view of Sotomayor theorem, if in addition to the above the following holds $W^{T}\left[D^{2} f\left(E_{0}, \alpha^{*}\right),(K, K)\right] \neq 0$, then system (1) possesses a transcritical bifurcation but no pitchfork bifurcation appears. Now since we get that:

$D^{2} f\left(E_{0}, \alpha^{*}\right),(K, K)=\left[\begin{array}{c}-2 \alpha^{*} z q k_{3}^{2} \\ 2 \alpha^{*} z q k_{3}^{2}+2 a b q^{2} k_{3}^{2} \\ -2 a b q^{2} k_{3}^{2}\end{array}\right]$,thus it followes that

$$
W^{T}\left[D^{2} f\left(E_{0}, \alpha^{*}\right),(K, K)\right]=2 q\left(\alpha^{*} z+\right.
$$

$a b q) k_{3}^{2} w_{2} \neq 0$. Therefore system (1) has a transcritical bifurcation at $E_{0}$ with $\alpha=\alpha^{*}$ provided that condition (13) holds.

Now it is well known that, the three dimensional dynamical system undergoes a Hopf bifurcation if and only if there is a complex conjugate eigenvalue, say $\lambda_{i}=\rho_{1} \mp \rho_{2}$, with third eigenvalue is real and negative (12), so that

$$
\rho_{1}\left(\ell^{*}\right)=0
$$

$$
\left.\frac{d \rho_{1}}{d \ell}\right|_{\ell=\ell^{*}} \neq 0
$$

where $\ell=\ell^{*}$ is a specific general parameter. This is an equivalent to that system (1) undergoes a Hopf bifurcation around the endemic equilibrium point if and only if the coefficient's of the characteristic polynomial given by (10b) satisfy that $\Omega_{i}>$ $0 ; \forall i=1,2,3$ with $\Omega_{1}\left(\ell^{*}\right) \Omega_{2}\left(\ell^{*}\right)=\Omega_{3}\left(\ell^{*}\right)$ (i.e. $\left.\Delta\left(\ell^{*}\right)=0\right)$ such that $\left.\frac{d \Delta}{d \ell}\right|_{\ell=\ell^{*}} \neq 0$. Since the condition (9) that guarantees $\Omega_{i}>0 ; \forall i=1,2,3$ is the same condition which guarantees $\Delta>0$, therefore there is no possibility to have a Hopf bifurcation for system (1).

\section{Numerical Analysis and Discussion}

In this section, the global dynamics of system (1) is investigated numerically for different sets of initial values and different sets of parameters values. The objectives of such investigation are to determine the effect of varying the parameters values and confirm our obtained results. It is observed that, for the following biologically feasible set of hypothetical parameters values:

$$
\begin{aligned}
A & =50, \delta_{0}=0.9, \alpha_{1}=0, \alpha=0.0001, \delta_{1}=0.2, \\
\delta_{2} & =0.2, \delta_{3}=0.4, a=4, b=0.2
\end{aligned}
$$

The basic reproduction number is $R_{0}=1.25$ and both the equilibrium points are exist. However the trajectories starting from different initial points approach asymptotically to the unique endemic equilibrium point $E_{1}=(113.74,31.36,42.14)$ as shown in the phase portrait given in Fig. (1) and their time series given in Fig. (2).

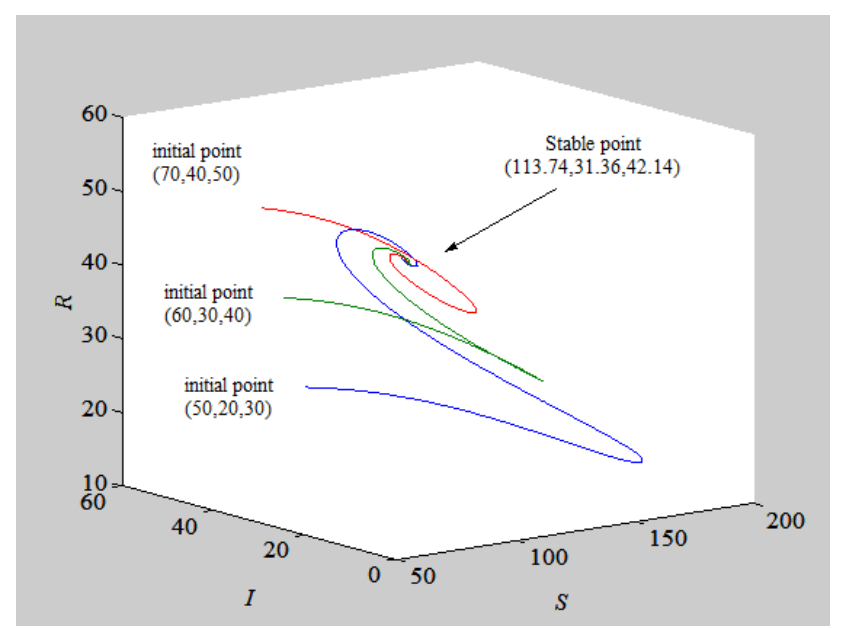

Figure 1. Globally asymptotically stable endemic equilibrium point of system (1) for the data in (15). 
Clearly these figures confirm the analytical results given by theorem (2) and theorem (5). However in case of losing the existence of disease free equilibrium point and the system has only endemic equilibrium point that will occur for

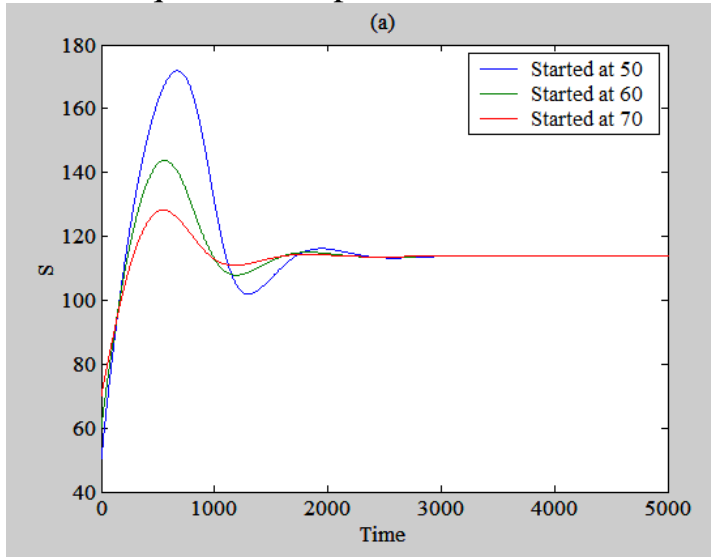

(b)

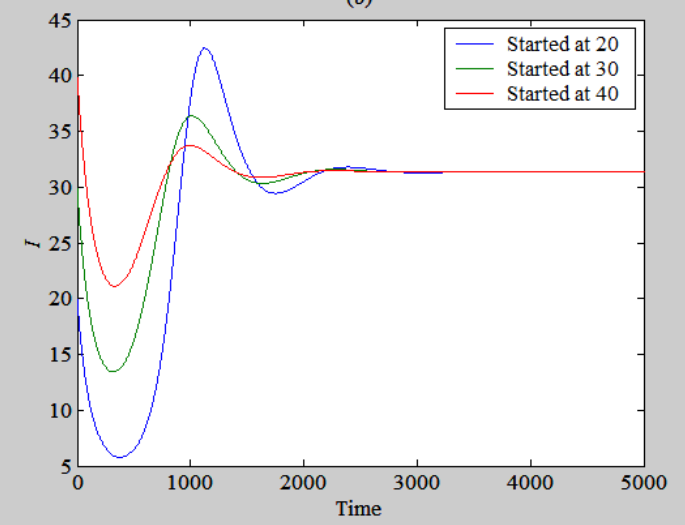

(c)

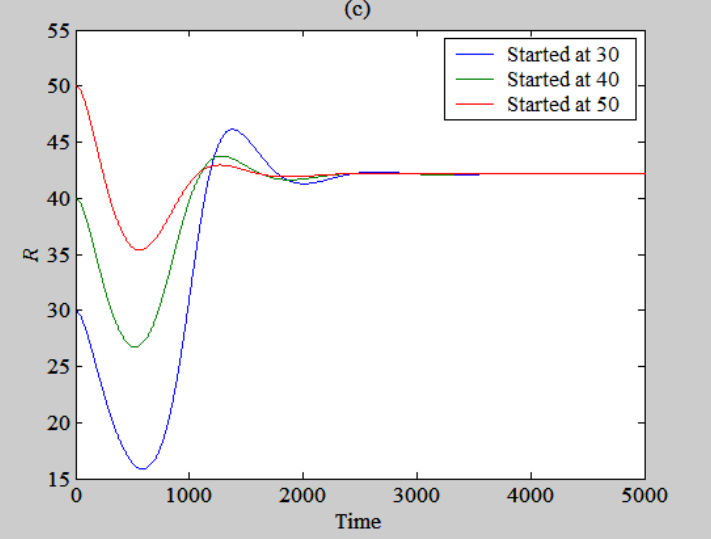

Figure 2. Time series of the trajectories given in Figure (1). (a) The trajectories of $S$ as a function of time. (b) The trajectories of $I$ as a function of $\alpha_{1}>0$, It is observed that the system has only point attractor and approaches asymptotically to endemic equilibrium point as shown in Fig. (3) for the data (15) with $\alpha_{1}=0.1$.

time. (c) The trajectories of $R$ as a function of time.

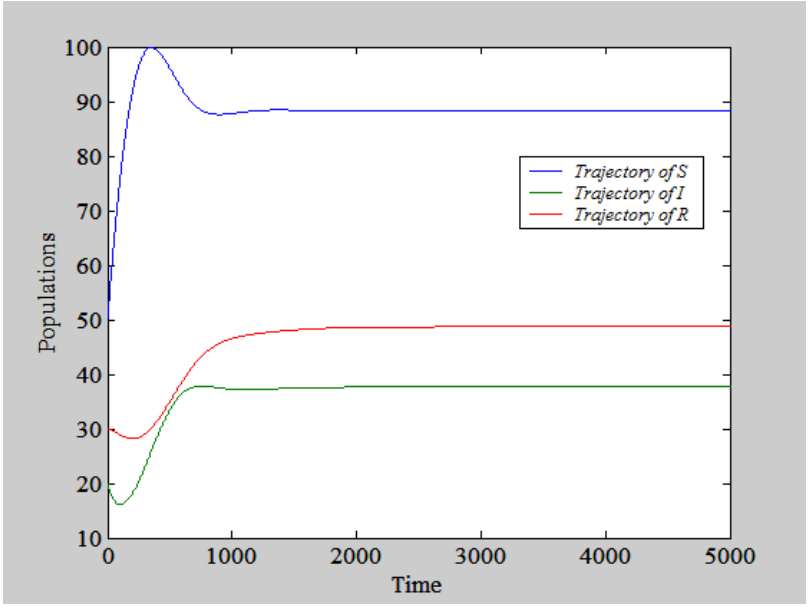

Figure 3. The trajectory of system (1) that approaches asymptotically to $E_{1}=(88.28,37.67,48.7)$ for the data $(15)$ with $\alpha_{1}=0.1$.

Now the effect of varying each parameter on the dynamical behavior of system (1) is investigated numerically for the data (15) in two cases: where there is no indirect incidence rate $\left(\alpha_{1}=0\right)$, and where there is an indirect incidence rate (say at $\alpha_{1}=0.01$ ). It is observed that as recruitment rate is $<40$, then $R_{0}<1$ and hence the trajectory of system (1) approaches asymptotically to the disease free equilibrium point as shown in the typical figure given by Fig. (4a) for the data (15) with $A=35$. However for the data given by (15) with $A=35$ and $\alpha_{1}=0.01$ the trajectory of system (1) still approaches asymptotically to endemic equilibrium point as shown in Fig. (4b) 

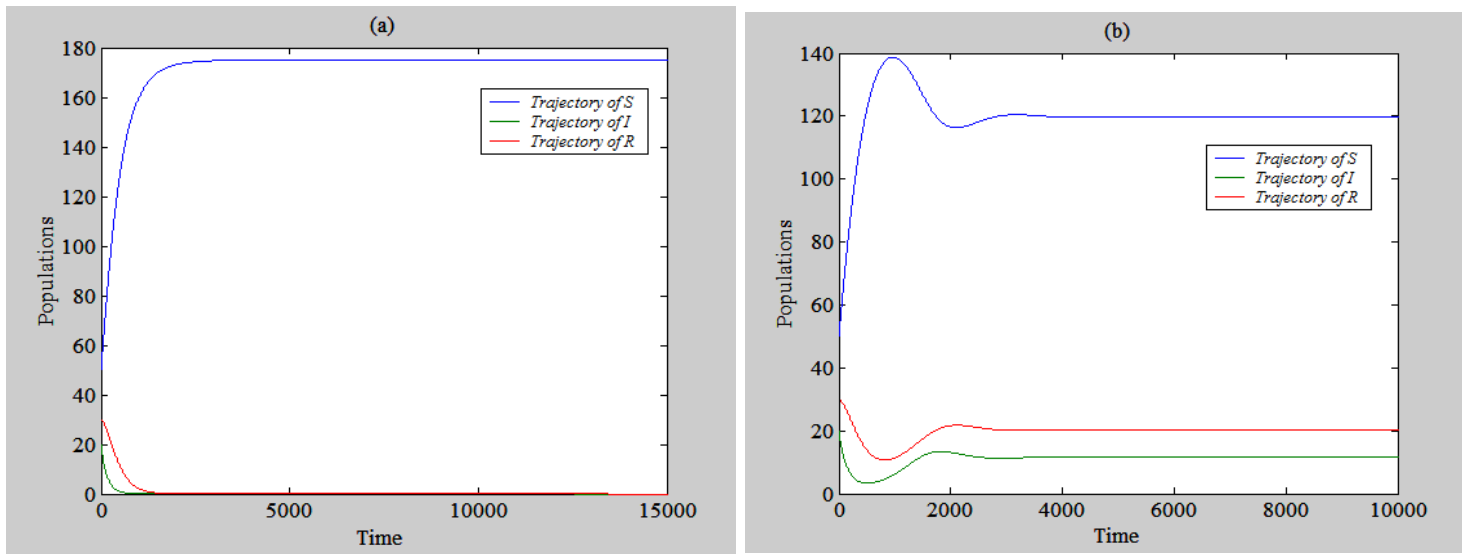

Figure 4. The trajectory of system (1). (a) System approaches to $E_{0}=(175,0,0)$ for data (15) with $A=35$. (b) System approaches to $E_{1}=(119.77,11.62,20.36)$ for data (15) $A=35$ and $\alpha_{1}=0.01$.

Obviously, for the data used in Fig. (4a) the basic reproduction number has the value $R_{0}=$ 0.875 and hence the solution approaches asymptotically to disease free equilibrium point as proved in theorem (2). However the solution of system (1) approaches asymptotically to the unique endemic equilibrium point as shown in Fig. (4b) even when $R_{0}=0.875$ due to the disappearances of the disease free equilibrium point, $\alpha_{1}>0$, and the bounded system (1) has no periodic dynamics. On the other hand, for all $A>40$ the value of $R_{0}>1$ and the solution of system (1) still

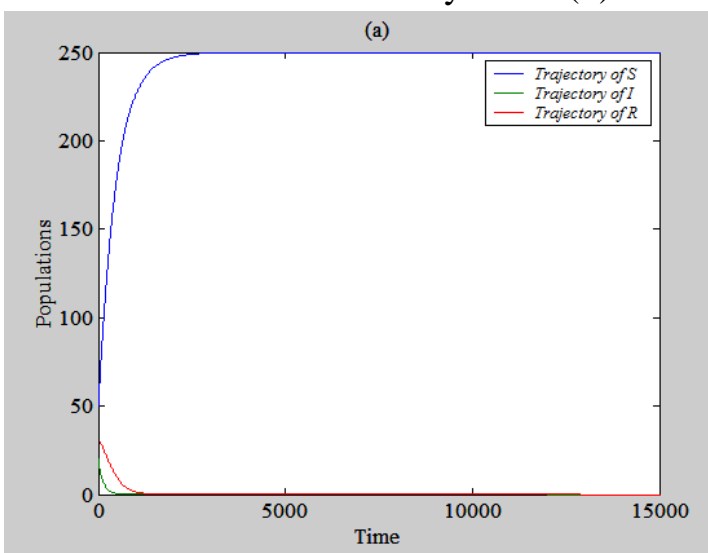

approaches to the endemic equilibrium point in both the cases. Moreover its observed that as the direct incidence rate $\alpha<0.008$, then $R_{0}<1$ and hence the trajectory of system (1) approaches asymptotically to the disease free equilibrium point as shown in the typical figure given by Fig. (5a) for the data (15) with $\alpha=0.007$. However for the data given by (15) with $\alpha=0.007$ and $\alpha_{1}=0.01$ the trajectory of system (1) still approaches asymptotically to endemic equilibrium point as shown in Fig.(5b).

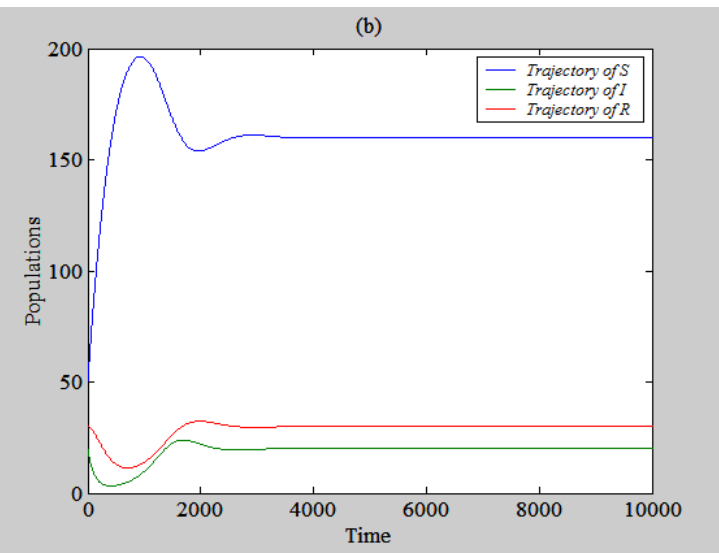

Figure 5. The trajectory of system (1). (a) System approaches to $E_{0}=(250,0,0)$ for data (15) with $\alpha=0.007$. (b) System approaches to $E_{1}=(160,20,30)$ for data (15) $\alpha=0.007$ and $\alpha_{1}=0.01$.

Clearly, for the data used in Fig. (5a), again the basic reproduction number has the value $R_{0}=$ 0.875 and hence the solution approaches asymptotically to disease free equilibrium point as proved in theorem (2). However the solution of system (1) approaches asymptotically to the unique endemic equilibrium point as shown in Fig. (5b) even when $R_{0}<1$ due to the disappearance of the disease free equilibrium point, $\alpha_{1}>0$, and the bounded system (1) has no periodic dynamics. Further for all $\alpha>0.008$ the value of $R_{0}>1$ and thus the solution of system (1) still approaches to the endemic equilibrium point in both the cases. Further, investigation of the effect of varying other parameter have been done and the observed results are shown in the following table. 
Table 1. The dynamical behavior of system (1) as varying of one variable with the other parameters as in (15)

\begin{tabular}{cccc}
$\begin{array}{c}\text { The range of } \\
\text { varying parameter }\end{array}$ & $\begin{array}{c}\text { The range } \\
\text { of } \boldsymbol{R}_{\mathbf{0}}\end{array}$ & $\begin{array}{c}\text { Dynamical behavior of } \\
\text { system }(\mathbf{1}) \text { where } \boldsymbol{\alpha}_{\mathbf{1}}=\mathbf{0}\end{array}$ & $\begin{array}{c}\text { Dynamical behavior of system (1) } \\
\text { where } \boldsymbol{\alpha}_{\mathbf{1}}=\mathbf{0 . 0 1}\end{array}$ \\
\hline$\delta_{0}<0.2445$ & $R_{0}>1$ & $E_{1}$ is asymptotically stable & $E_{1}$ is asymptotically stable \\
$\delta_{0}>0.25$ & $R_{0}<1$ & $E_{0}$ is asymptotically stable & \\
$\delta_{1}<0.9$ & $R_{0}>1$ & $E_{1}$ is asymptotically stable & $E_{1}$ is asymptotically stable \\
$\delta_{1}>0.9$ & $R_{0}<1$ & $E_{0}$ is asymptotically stable & \\
$\delta_{2}<0.9$ & $R_{0}>1$ & $E_{1}$ is asymptotically stable & $E_{1}$ is asymptotically stable \\
$\delta_{2}>0.9$ & $R_{0}<1$ & $E_{0}$ is asymptotically stable & \\
$\delta_{3}>0$ & $R_{0}>1$ & $E_{1}$ is asymptotically stable & $E_{1}$ is asymptotically stable \\
$a<1.5$ & $R_{0}>1$ & $E_{1}$ is asymptotically stable & $E_{1}$ is asymptotically stable \\
$a>1.5$ & $R_{0}<1$ & $E_{0}$ is asymptotically stable & \\
$b>0$ & $R_{0}>1$ & $E_{1}$ is asymptotically stable & $E_{1}$ is asymptotically stable \\
\hline
\end{tabular}

According to the above table, it is observed that varying the parameters that stand for partial temporary immunity rate $\delta_{3}$ and the resource limitation $b$ don't change the value of $R_{0}$, as they do not appear in the form of it, and hence they do not have a qualitative change on the dynamical behavior of the system rather than that they have quantitative change on the population size of each compartments of the system. All other parameters have a bifurcation point at $R_{0}=1$ and hence they confirm our analytical results regarding the stability and bifurcation analysis in case of there is no indirect incidence rate. However adding indirect incidence rate to the system will causes the disappearance of the disease free equilibrium point from the system and hence the system will always approach to the endemic equilibrium point. Finally, the system has only one type of attractor that is a stable point and there is no periodic dynamics. Finally in order to control the disease the value of natural death rate $\delta_{0}$, disease death rate $\delta_{1}$, natural recovery rate $\delta_{2}$ and maximum treatment rate $a$ should be kept large enough.

\section{Conflicts of Interest: None.}

\section{References:}

1. Murray JD. Mathematical biology. Berlin Heidelberg. USA: Springer-Verlag; 2003.

2. Bunimovich-Mendrazitsky S, Stone L. Modeling polio as a disease of development. J. Theo. Biol., 2005; 237(3): 302-315.
3. D'Onofrio. A Pulse Vaccination Strategy in the SIR Epidemic Model, Global Asymptotic Stable Eradication in Presence of Vaccine Failures, j.mcm, 2002; 36: 473-489.

4. Dubey B, Dubey P, Dubey US. Dynamics of an SIR Model with Nonlinear Incidence and Treatment Rate, AAM ,2015; 10 (2): 718 - 737.

5. Taylor M L, Carr T W. An SIR epidemic model with partial temporary immunity modeled with delay, J. Math. Biol., 2009; 39 (6): 841-880.

6. Sahu G P, Dhar J. Analysis of an SVEIS epidemic model with partial temporary immunity and saturation incidence rate, Appl. Math. Model, 2012; 36 (3): 908-923.

7. Naji RK, Hussien RM. The Dynamics of Epidemic Model with Two Types of Infectious Diseases and Vertical Transmission, J App Math, 2016; 16 pages.

8. Adebimpe O, Bashiru KA, Ojurongbe TA. Stabillity analysis of an SIR epidemic model with nonlinear incidence rate and treatment. Open Journal of Modelling and Simulation, 2015; 3: 104-110.

9. Anderson B, Jackson J, Sitharam M. Descartes' Rule of Signs Revisited. Am Math Mon, 1998; 105 (5): 447-451.

10. Van den Driessche P, Watmough J. Reproduction numbers and sub-threshold endemic equilibria for compartmental models of disease transmission, Math Biosci, 2002; 180: 29-48.

11. Perko L. Differential Equation and Dynamical Systems, 3rd edition. New York NY, USA: Springer; 2001.

12. Sarkar M, Das T, Mukherjee RN. Bifurcation and Stability of Prey-Predator Model with BeddingtonDeAngelis Functional Response, AAM, 2017; 12 (1): 350-366. 
تحليل المناعة المؤقته الجزئية لنموذج SIR للأمراض الوبائية بمعامل علاج غير خطي رائد كامل ناجي 2

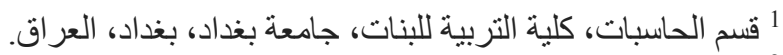
2² قسم الرياضيات، كلية العلوم، جامعة بغداد، بغداد، العراق.

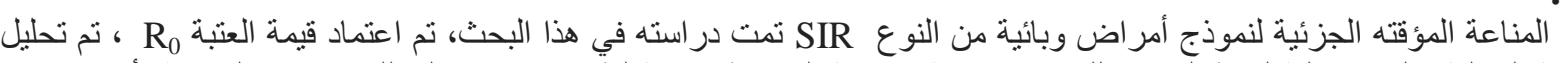

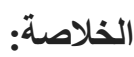

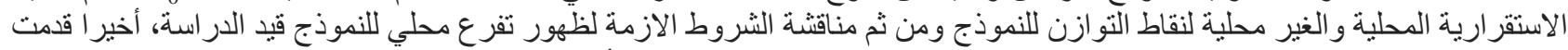

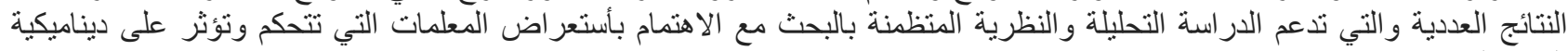

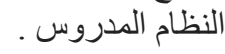
الكلمات المفتاحية : مناعة مؤقته جزئية، معامل علاج غير خطي، نموذج SIR الوبائي. 